\title{
Intestinal Bile Acid Malabsorption in Cystic Fibrosis: a Primary Mucosal Cell Defect
}

\author{
JOSEPH D. FONDACARO ${ }^{(35)}$, JAMES E. HEUBI, AND FRANK W. KELLOGG \\ Departments of Physiology and Pediatrics, University of Cincinnati College of Medicine, Cincinnati, Ohio and \\ Children's Hospital Clinical Research Center, Cincinnati, Ohio USA
}

\section{Summary}

Bile acid malabsorption in cystic fibrosis reduces intraluminal bile acid concentration and may impair fat absorption. The cause of this malabsorption is unknown but it is believed due to intraluminal inhibition of uptake by undigested dietary nutrients. The purpose of this study was to determine the bile acid absorptive capability of cystic fibrosis intestine in a physiologic environment. Direct ileal mucosal taurocholic acid uptake was examined in vitro in seven patients with cystic fibrosis, and seven children and adolescents with ileostomies as controls. Jejunal uptake was studied in five normal individuals. A Crosby-Kugler biopsy capsule was used to obtain all tissues. Tissue was incubated in Krebs buffer, $10 \mathrm{mM}$ glucose, and taurocholic acid at $0.1,1.0$ and $10.0 \mathrm{mM}$ with shaking at $37^{\circ} \mathrm{C}$. Significant reduction of taurocholic acid uptake was present in every cystic fibrosis patient with mean uptake rates being $24 \%, 38 \%$, and $29 \%$ of control ileum, respectively, at the three concentrations. Values paralleled those for passive jejunal taurocholic acid uptake in controls. These data illustrate a marked reduction in taurocholic acid uptake capability of cystic fibrosis ileal mucosa and may indicate a cellular defect suggestive of a primary lesion in this disease.

\section{Speculation}

Results of the present study suggest that bile acid malabsorption in cystic fibrosis may be due to a primary functional defect of the ileal mucosal cells in this disease. This is demonstrated in vitro by the reduced uptake rates for taurocholic acid by ileal mucosa taken from cystic fibrosis patients when compared to controls. Furthermore, the taurocholic acid uptake rate in cystic fibrosis ileal mucosa are comparable to that of jejunal mucosa from health volunteers. This suggests that active transport mechanisms for bile acid absorption are absent in cystic fibrosis intestine. Although these studies do not impugn an intraluminal mechanism, they point to a primary functional ileal mucosal cell defect as the pathophysiologic mechanism of bile acid malabsorption in cystic fibrosis.

Cystic fibrosis (CF) is an autosomal, recessively transmitted disease affecting multiple organs including the mucous glands of the tracheobronchial tree, the exocrine pancreas, and the gastrointestinal mucosa (15). Clinically significant intestinal malabsorption of various substrates including bile acids is a consistent finding in CF $(1,3,5,12,16,21,25,28,30,33)$.

Several studies have been carried out attempting to define the pathophysiology and the severity of bile acid malabsorption in CF. Total bile acid pool size is contracted in CF patients unsupplemented with pancreatic enzymes, which reverses with supplementation (30). Fecal bile acid loss in CF, which directly correlates with the degree of pancreatic insufficiency (32), can be excessive due to interruption of the normal enterohepatic circulation of bile acids.

There are several theories regarding the factor or factors that might account for the inhibition of bile acid absorption in CF.
The most widely accepted view suggests that undigested dietary nutrients (present intraluminally due to pancreatic insufficiency) in some way bind or sequester bile acids, thus preventing normal absorption $(3,15,21,33)$; however, definitive studies have not been reported and the precise mechanism remains conjectural.

The present study was designed to determine if ileal mucosa from CF patients, when removed from the abnormal intraluminal environment of the CF intestine, has the normal capacity to absorb bile acid in vitro. Results from such experiments will either suggest that the bile acid malabsorption in CF is caused by an intraluminal event or will point toward a basic cellular defect for bile acid absorption in the ileal mucosa.

\section{MATERIALS AND METHODS}

Patients. Seven patients (two males, five females) with cystic fibrosis, $\mathrm{CF}(\mathrm{Il})$, age $4-8 / 12$ years to 25 years (mean 16.7 years) were studied. All had sweat chloride concentrations exceeding 80 $\mathrm{mEq} /$ liter on repeated tests, pulmonary disease confirmed by pulmonary function studies and chest radiographs, and pancreatic insufficiency defined as absent stool tryptic activity and improvement of diarrhea with pancreatic enzyme supplements. All patients were hospitalized for aggressive pulmonary toilet and broad spectrum intravenous antibiotics including a semisynthetic penicillin and an aminoglycoside. Before discharge, during a 7-10 day course of antibiotics, the present study was performed. All patients were below the 5 th percentile for weight and/or height.

The first control group, C(I1), consisted of seven patients (three males, four females) ages $6 / 12$ to 27 years (mean 11.1 years) who had well adapted ileostomies (at least 12 months postresection) after total colectomy for ulcerative colitis (six patients) or Crohn's colitis (one patient). All were in excellent health, and all mucosae were histologically normal. We believe this is the most reasonable control group to study because peroral ileal biopsy in normal healthy children of this age is neither feasible nor ethical.

Four females and one male, ages $26-43$ years (mean 31 years), all in good health and with no gastrointestinal symptoms, were studied as a jejunal control group, $\mathrm{C}(\mathrm{Je})$. All mucosae were histologically normal.

Informed consent was obtained from either the patient or his/ her parents before entering the study. Protocol for the study was approved by the Committee on Human Research of the University of Cincinnati, College of Medicine, and by the Committee on Research Involving Human Subjects of the Children's Hospital Medical Center, Cincinnati, Ohio.

Biopsy procedure. Peroral biopsies were taken from the ileum in seven $C F$ patients and from the jejunum in the five normal volunteers using a $3.0 \mathrm{~mm}$ port Crosby-Kugler biopsy capsule. Proximal jejunal biopsies were performed as previously described (23). Ileal biopsies were performed according to the following procedure. After an overnight fast, an Argyl nasogastric tube was passed through the nose and the tip retrieved through the month with a McGill forceps. The proximal end of a polyethylene tube (180-240 cm in length) attached to the biopsy capsule, was then 
pulled back through the nose. The patients swallowed the capsule with the tubing extended out through the nose. Passage of the capsule through the pylorus was facilitated by patient positioning. The patient was allowed to eat and the capsule moved distally with peristalsis. The position of the capsule was verified periodically using abdominal $x$-ray with air as contrast medium. In no case did the capsule take longer than $48 \mathrm{~h}$ to arrive at the terminal ileum. In most cases after $24 \mathrm{~h}$ the capsule had advanced into the ascending colon in which case it was gently pulled back through the ileocecal valve for biopsy of the ileum. Biopsies were taken within $8-10 \mathrm{~cm}$ of the ileocecal valve. In all cases capsule retrieval time was under $90 \mathrm{sec}$. The seven control patients with ileostomies (cutaneous or in ano) were biopsied with the capsule through the ostomy to a distance of $6-8 \mathrm{~cm}$ proximal to the skin opening. In all studies the biopsy specimens were placed in a Petri dish containing Krebs buffer and viewed under a stereomicroscope to determine its gross appearance. A portion of the tissue was taken for transmission electron microscopic study. The remaining portion of the mucosa was divided into three equal sections for the absorption study.

Incubation procedure and analysis. Intestinal mucosal uptake of taurocholic acid (TC) was measured using a modification of the villus technique $(7,8)$. Sections of intestinal biopsy samples were quickly transferred to incubation vessels which consisted of $50 \mathrm{ml}$ beakers containing $4.9 \mathrm{ml}$ of Krebs buffer ( $\mathrm{pH} \mathrm{7.4)}$ at $37^{\circ} \mathrm{C}$, covered with a rubber stopper fitted with glass tubing for gassing with $5 \% \mathrm{CO}_{2}$ in $\mathrm{O}_{2}$. The tissue was allowed to reach temperature equilibration for $30 \mathrm{sec}$ while the vessels were shaken at $140 \mathrm{c} /$ min in a Dubnoff incubation bath. At this time $100 \mu \mathrm{l}$ of a concentrated TC solution with $\left[{ }^{3} \mathrm{H}\right]-\mathrm{TC}$ (New England Nuclear, Boston, MA) was pipetted into the vessel so that the final concentration of TC was either $0.1,1.0$, or $10.0 \mathrm{mM}$. After a 2-min incubation, tissue samples were recovered by filtration through a nylon screen, quickly rinsed with cold saline and placed in a small vial containing $1.0 \mathrm{ml}$ of iced Krebs buffer. The vials containing the tissue were immediately frozen in dry-ice acetone and lyophilized overnight. Afterwards, the pieces were tapped free of freeze dried salts, weighed, and dissolved in $1.0 \mathrm{ml}$ of $90 \%$ Soluene-350 (Packard, Downers Grove, IL) at $60^{\circ} \mathrm{C}$. Villi were not removed as outlined in the original description of this technique, because no substantial amount of submucosa and no visceral muscle were present. After addition of $10 \mathrm{ml}$ of counting solution (ACS, Amersham, Arlington Heights, IL) the radioactivity was determined (Beckman, LS-333, Fullerton, CA) in these epithelial cell rich tissue samples, in $0.5 \mathrm{ml}$ of the bathing medium and in $0.5 \mathrm{ml}$ of a standard for each concentration of TC run concurrently. Using $\left[{ }^{3} \mathrm{H}\right]$-insulin, we have determined the distribution of extracellular space to be less than $0.1 \%$.

Using a specific activity in $\mathrm{cpm} / \mu$ mole of TC determined from the standards and appropriate calculations using tissue weight (g) and time (min), uptake of TC is expressed as $\mu$ moles $\cdot \mathrm{g}$ (dry weight $)^{-1} \cdot \min ^{-1}$. Results are presented as the mean \pm 1 S.E.

Data from all experiments in each group was statistically evaluated using the Student's $t$ test for pooled observations. The significance of these changes was assumed at a $P$ value less than 0.05 .

Electron microscopy. A representative section of all biopsy tissue was prepared for transmission electron microscopy by fixation in $1 \%$ osmium alone and $3 \%$ glutaraldehyde followed by "postfixation" in $1 \%$ osmium. Reagents were prepared and stored as previously described (23).

Fecal bile acid excretion. Stool (72-h collections) frozen at $0^{\circ} \mathrm{C}$ was thawed and homogenized in water. Bile acids were extracted and quantitated by a modification of the method of Weber et al. (31) using spectrophotofluorometry as previously described (22). Cholic 2,4- $\left[{ }^{3} \mathrm{H}\right]$ acid (New England Nuclear, Boston), added to freshly thawed stool, was used as an internal standard. Standard concentration curves were performed daily; the coefficient of variation was $1.87 \%$. Analyses were performed on stool samples from five $C F$ patients and five controls and the results were statistically compared using the Student's $t$ test for pooled observations.

\section{RESULTS}

The rate of mucosal uptake of TC was studied at three different initial concentrations, i.e., $0.1,1.0$, and $10.0 \mathrm{mM}$ in tissue taken from the three groups of patients. The data shown in Figure 1 summarizes the TC uptake rates in the three groups of tissue when the incubation medium contained an initial TC concentration of $0.1 \mathrm{mM}$. TC uptake in the ileum of the control group, C(I1), was $1.42 \pm 0.28 \mu$ moles $\cdot g(\text { d.w. })^{-1} \cdot \min ^{-1}$. In the CF (II) group, TC uptake was dramatically reduced to only $24 \%$ of C(II), $(0.34 \pm$ $0.06)$. This value was not different from that $(0.29 \pm 0.05 \mu$ moles - $\left.g(\text { d.w. })^{-1} \cdot \min ^{-1}\right)$ observed for jejunal uptake of TC in the normal volunteers.

Similar results were observed with tissue incubated at an initial TC concentration of $1.0 \mathrm{mM}$ (Fig. 2). The C(I1) tissue absorbed $6.36 \pm 1.33 \mu$ moles $\cdot \mathrm{g}(\mathrm{d} . \mathrm{w} .)^{-1} \cdot \mathrm{min}^{-1}$ of TC whereas the CF(I1) tissue absorbed only $38 \%$ of this value $(2.41 \pm 0.34)$. This latter rate was not significantly different from the $\mathrm{C}(\mathrm{Je})$ rate of $3.58 \pm$ 0.75 .

Likewise, qualitatively similar results were observed when intestinal mucosae were incubated in TC at $10.0 \mathrm{mM}$ (Fig. 3). In $\mathrm{CF}(\mathrm{Il})$ tissue, TC uptake rate was $22.17 \pm 3.04 \mu$ moles $\cdot \mathrm{g}(\mathrm{d} . \mathrm{w} .)^{-1}$ - min $^{-1}$ which represents only $29 \%$ of the C(I1) tissue value (76.21 \pm 19.30 ). Again the rate of TC uptake in CF(II) paralleled the $27.90 \pm 5.42 \mu$ moles $\cdot g(\text { d.w. })^{-1} \cdot \min ^{-1}$ value observed in the $\mathrm{C}(\mathrm{Je})$ group.

Electron microscopic studies of ileal mucosa taken from $\mathrm{CF}$

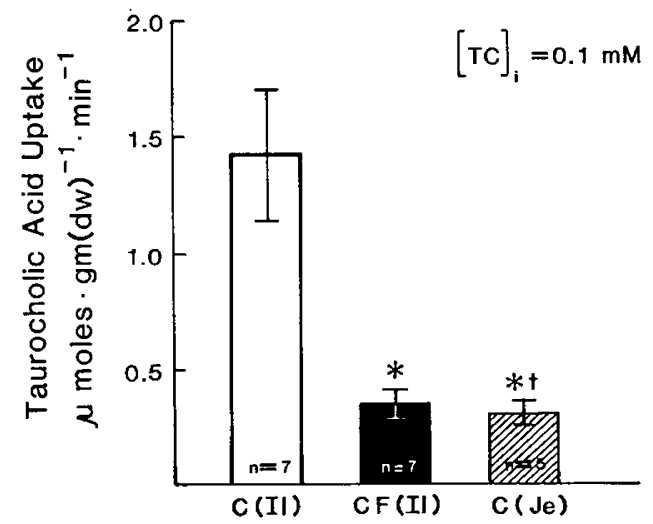

Fig. 1. Comparison of taurocholic acid (TC) uptake rates in mucosa from ileum of cytsic fibrosis patients, $\mathrm{CF}(\mathrm{H1})$, ileum of controls, $\mathrm{C}(\mathrm{Il})$ and jejunum of controls, $\mathrm{C}(\mathrm{Je})$. Initial (TC) concentrations, $0.1 \mathrm{mM} ;{ }^{*} P<$ 0.001 vs $\mathrm{C}(\mathrm{I} 1)$; and ${ }^{+}$No significant difference vs $\mathrm{CF}(\mathrm{I} 1)$.

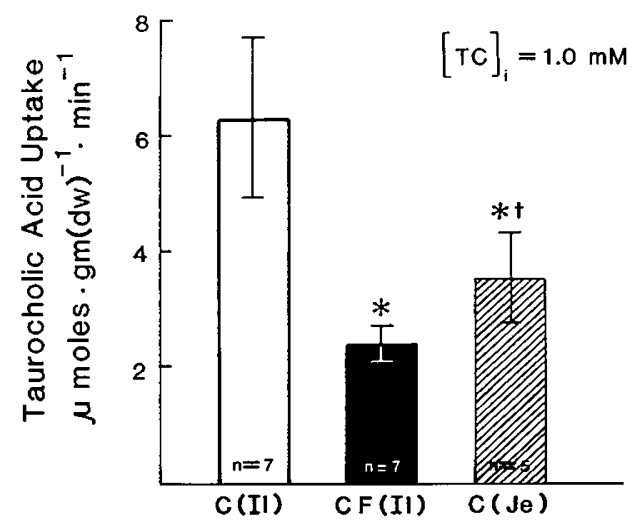

Fig. 2. Comparison of taurocholic acid (TC) uptake rate in mucosa from $\mathrm{CF}(\mathrm{II}), \mathrm{C}(\mathrm{Il})$, and $\mathrm{C}(\mathrm{Je})$. Initial TC concentration, $1.0 \mathrm{mM} ;{ }^{*} P<$ 0.05 vs $\mathrm{C}(\mathrm{II})$; and ${ }^{+}$No significant difference $v$ s $\mathrm{CF}(\mathrm{II})$. 
patients indicated that the brush border membrane of these tissues was normal and indistinguishable from the same tissue taken from controls. Fig. 4 presents representative transmission electron micrographs of ileal brush border from a control subject (left panel) and from a CF patient (right panel). There was no appreciable mucus or other abnormal substance adhering to the microvilli. Furthermore, there was no structural evidence of any cytological disease in the CF mucosal cells. Thus, the CF tissue used in this study was considered to have normal structure and glycocalyx.

Fecal bile acid levels were determined in five CF patients who

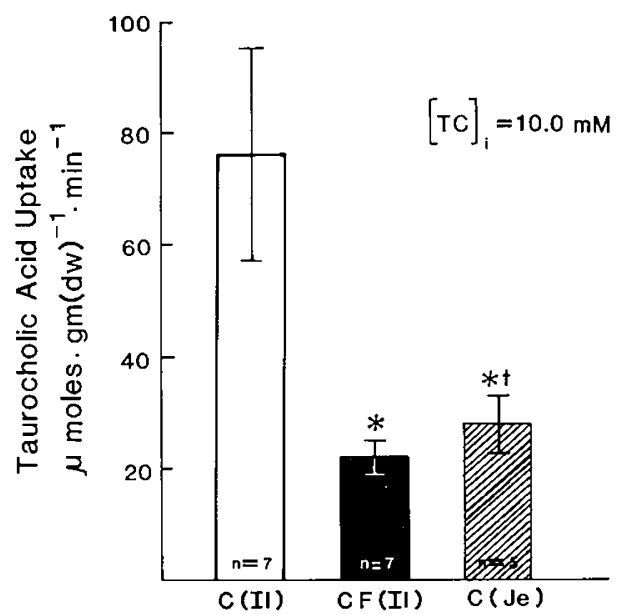

Fig. 3. Comparison of taurocholic acid (TC) uptake rates in mucosa from $\mathrm{CF}(\mathrm{Il}), \mathrm{C}(\mathrm{Il})$, and $\mathrm{C}(\mathrm{Je})$. Initial TC concentration, $10.0 \mathrm{mM} ;{ }^{*} P<$ 0.05 vs $\mathrm{C}(\mathrm{Il})$; and ${ }^{+}$No significant difference vs $\mathrm{CF}(\mathrm{I} 1)$.

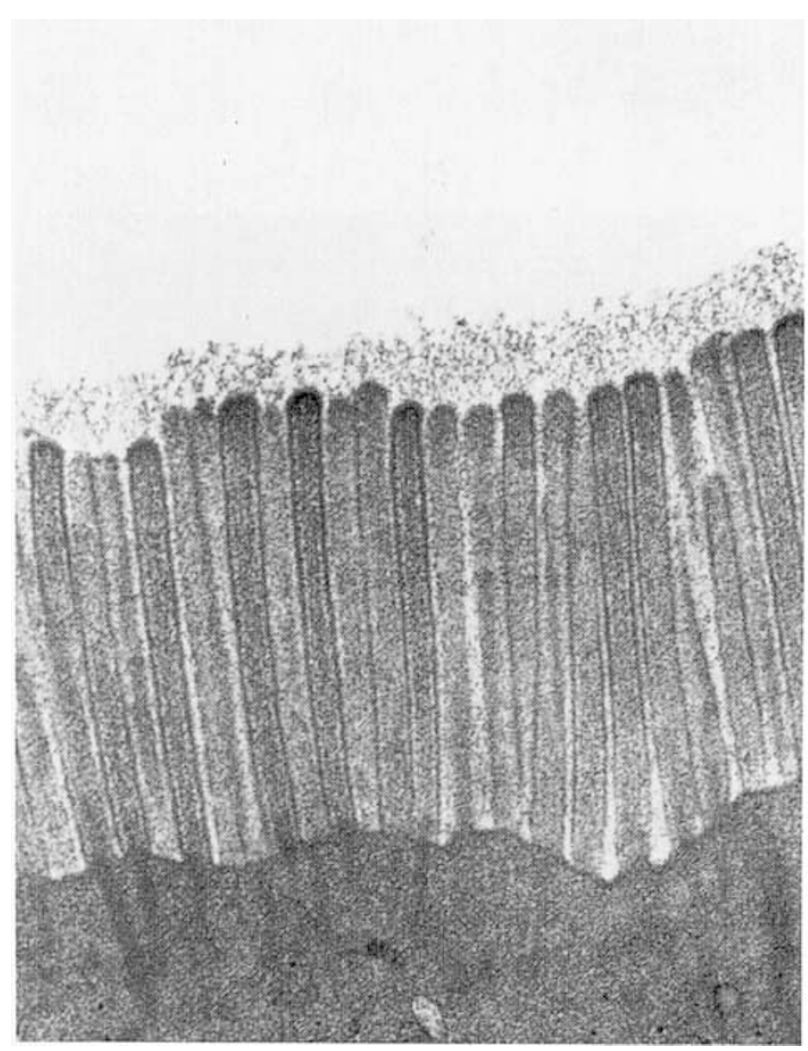

A were included in the absorption study and five normal controls. Results are shown in Table 1. The mean total fecal bile acid excretion $\left(\mathrm{mg} / \mathrm{m}^{2} /\right.$ day) in the CF patients was significantly elevated over that of the control group $(P<0.05)$.

\section{DISCUSSION}

Bile acids are discharged into the small intestine following a meal stimulus. These substances are essential for the process of dietary lipid digestion and absorption and are also thought to enhance pancreatic lipase activity and facilitate intramucosal triglyceride reesterification (17). Bile acids are reabsorbed along the length of the intestinal tract and returned to the liver for recycling with only a small amount $(2-5 \%)$ of the total bile acid pool excreted during each of the 6-10 cycles daily. The terminal ileum contains a highly efficient active transport mechanism for bile acids and is the primary site for absorption of these substances (2, $8,17,29$ ). Although other areas of the small intestine allow passive absorption of bile acids, interruption of active ileal transport, as may occur with ileal disease or surgical resection, produces diarrhea, steatorrhea, increased bile acid excretion and a reduction in the total bile acid pool.

Table 1. Fecal bile acid levels (mean $\pm S . E$.) of five CF patients and five controls (with ileostomies) that were included in the absorption study. Values are expressed as $\mathrm{mg} / \mathrm{m}^{2} /$ day

\begin{tabular}{ccc}
\hline Controls & CF \\
\hline $90.8 \pm 21.9$ & $(P<0.05)^{1}$ & $214.0 \pm 48.4$ \\
\hline
\end{tabular}

' Statistical comparison performed using the Student's $t$-test for pooled observations, with significance accepted at $P$ less than 0.05 .

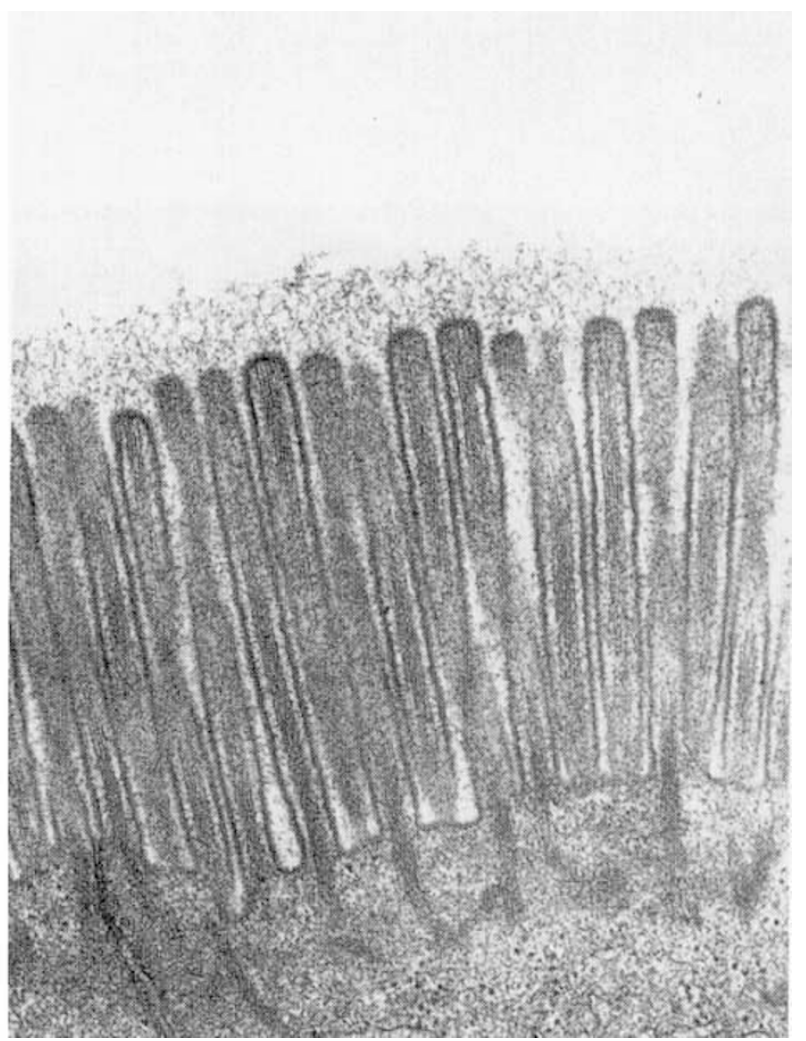

Fig. 4. Transmission electron micrographs of brush border membrane of biopsy tissue from control ileum (left panel) and from cystic fibrosis ileum (right panel) $(\times 21,500)$. 
Bile acid malabsorption is a well recognized sequela of cystic fibrosis. Many investigators support the proposal that undigested dietary substrates are the cause of bile acid malabsorption $(3,15$, 21,33 ), presumably by intraluminal binding or sequestration of bile acid molecules thereby inhibiting normal absorption. Recent animal studies from our laboratory suggest that only dietary triglyceride may adversely affect bile acid absorption and that carbohydrate and protein do not interfere with this process (9). Although treatment of CF patients with pancreatic supplements reduces excess bile acid excretion, fecal bile acid loss is not normalized $(30,32)$. Furthermore, bile acid malabsorption in some CF patients is so severe that it cannot be adequately accounted for solely by a simple intraluminal event. Recently Eklund et al. (6) have suggested that factors other than intraluminal binding of bile acids by unhydrolyzed nutrients must be considered to explain this malabsorption.

The results of the present study suggest that malabsorption of bile acids may be caused by a primary ileal mucosal cell defect in cystic fibrosis. This is evidenced by the fact that taurocholic acid uptake by ileal mucosa from CF patients, when placed in physiological medium, is not comparable to the uptake by the same tissue taken from control patients. Furthermore, the rate of uptake of taurocholic acid by CF ileal mucosa is comparable to the uptake rates seen in jejunal mucosa from normal volunteers. Becuase bile acid absorption occurs only by passive movement in the jejunum in vivo, this suggests that only passive, diffusional absorption is occurring in tissue which ordinarily should have active transport capabilities for bile acids.

It has been reported by Thomaidis and Arey (27) that patients with cystic fibrosis of the pancreas have severe involvement of the intestinal glands. This could lead to abnormal mucus accumulation along the intestinal microvilli and possible impairment of normal absorptive function; however, electron microscopic studies reported here clearly illustrate that brush border membranes of ileal tissue from $\mathrm{CF}$ patients was normal and void of mucus thus indistinguishable from control ileal brush border. Although Freye et al. (10) have reported the appearance of a "coarse fibrillar substance" in duodenal and jejunal biopsy specimens from CF patients, our studies indicate only a trace of such a substance in our ileal biopsies from these patients. It is unlikely that such a mechanism could impair bile acid absorption. Furthermore, close examination of the $\mathrm{CF}$ mucosal cells shows no structural evidence of cytologic disease, suggesting that a biochemical derangement is most likely responsible for our findings of reduced bile acid absorption

Antibiotic therapy may profoundly influence bile acid excretion in CF patients (24). Roy, et al. (24) have reported that although oral cloxacillin had no significant effect, triple IV therapy (cloxacillin, gentamicin and carbenicillin) markedly reduced fecal bile acid loss in CF patients. The CF patients in our study were on a semi-synthetic penicillin and an aminoglycoside which may produce different effects than those observed by Roy et al (24). Neither cultures of ileal discharge nor stool cultures were performed to answer this question. Nonetheless, fecal bile acid excretion remained increased in our patients despite antibiotics. In our $\mathrm{CF}$ patients, therefore, antibiotic therapy may have led to partial but incomplete improvement of their bile acid malabsorption.

In the present study, patients with well adapted ileostomies comprised the control group. As mentioned in "Materials and Methods," we feel this is the only reasonable and feasible group of patients to use as controls. Although adaptive changes in bile acid absorption in these patients might be expected, there is no data to support this hypothesis. Despite alterations in ileal bacterial flora (11), fecal bile acid excretion in ileostomy patients is within the normal range, providing a portion of the ileum has not been resected $(13,19)$. Because none of our control patients had ileal resection we feel confident of the adequacy of this group as controls for this study.

Although our findings do not disprove the intraluminal theory, they do support the concept that some basic physiological com- ponent of normal bile acid absorption by ileal mucosal cells is compromised in cystic fibrosis. This is similar to what has been observed for amino acid malabsorption in CF patients $(20,21)$. It is possible that the pathophysiologic mechanism underlying these patterns of malabsorption is a mucosal membrane defect, because other investigators have identified membrane dysfunctions in other CF tissues. For example, a recent study by Katz (14) has revealed a specific defect in $\mathrm{Mg}^{++}, \mathrm{Ca}^{++}$-ATPase of membranes prepared from CF fibroblasts and red blood cells. Also, alterations in peripheral glycoprotein, intrinsic protein, and alpha-L-fucosidase activity have been documented in CF fibroblast membranes by Scanlin and Glick (26). It is also possible that multiple pathophysiologic mechanisms underlie bile acid malabsorption in CF. For instance, studies by Mangos and McSherry (18) have demonstrated that high levels of sodium in sweat and saliva of CF patients may be due to the presence of a "sodium reabsorption inhibiting factor." Likewise, a serum factor from CF patients has been proposed by Conover et al. (4) to cause inhibition of ciliary movement in tracheal explant cultures, probably by altering ion fluxes across cell membranes. Because ileal bile acid transport is sodium coupled, these proposed "humoral" factors may adversely influence the bile acid transporting cells of the ileal mucosa by inhibiting sodium flux.

It may be that some or all of the pathophysiologic mechanisms just described contribute to some extent to the total bile acid malabsorption experienced by $\mathrm{CF}$ patients; however, our present study suggests that intestinal malabsorption of bile acids in $\mathrm{CF}$ results, at least in part, from a primary ileal cell defect in this disease.

\section{REFERENCES AND NOTES}

1. Balsam M. J., Holtzapple, P. G., Kaye, R., and Sewel, E. M.: Intestinal absorption of insulin in patients with fibrocystic disease. J. Pediatr., 79: 1011 (1971).

2. Bergstrom, S.: Metabolism of bile acids. Fed. Proc., 21: 28 (1962).

3. Bile Acid Malabsorption in Cystic Fibrosis. Nutr. Rev., 32: 232 (1974).

4. Conover, J. H., Conod. E. J., Gaerlan, P. F., and Bogart, H. I.: Calcium flux and cystic fibrosis. Lancet, I2: 1362 (1976).

5. Deren, J. J.. Arora, B., Toskes, P. P., Hansel, J., and Sibinga, M. S.: Malabsorption of crystalline vitamin $B_{12}$ in cystic fibrosis. N. Engl. J. Med., 288: 945 (1973).

6. Eklund, A., Norman, A., and Strandvik, B.: Excretion of bile acids in healthy children and children with cystic fibrosis. Scan. J. Clin. Lab. Invest., 40: 595 (1980).

7. Fondacaro, J. D., Nathan, P., and Wright. W. E.: Methionine accumulation in villi isolated from maturing rat intestine. J. Physiol., 241: 751 (1974).

8. Fondacaro, J. D. and Rodgers, J. B., Jr.: Characterization and effects of phospholipid on bile acid absorption by villi isolated from hamster small intestine. Am. J. Dig. Dis., 23: 12 (1978).

9. Fondacaro, J. D. and Wolcott, R. H.: Effects of dietary nutrients on intestinal taurocholic acid absorption. Proc. Soc. Exp. Biol. Med., 168: 276 (1981).

10. Freye, H. B., Kurtz, S. M., Spock, A., and Capp, M.: Light and electron microscopic examination of the small bowel of children with cystic fibrosis. J. Pediatr., 64: 575 (1964)

11. Gorbach, S. L., Nahas, L. and Weinstein, L.: Studies of intestinal microflora. IV The microflora of ileostomy effluent: a unique microbial ecology. Gastroenterology, 56: 874 (1967).

12. Harries, J. T. and Muller, D. P. R.: Absorption of different doses of fat soluble and water miscible preparations of vitamin $E$ in children with cystic fibrosis. Arch. Dis. Child., 46: 341 (1971).

13. Huibregtse, K., Hoek, F., Sanders, G. T. B., and Tytgat, G. N. J.: Bile acid metabolism in ileostomy patients. Euro. J. Clin. Invest., 7: 137 (1977).

14. Katz, S.: $\mathrm{Ca}^{++}$-transport and $\left(\mathrm{Mg}^{++}+\mathrm{Ca}^{++}\right)$-ATPase activity in cystic fibrosis patients. In: Sturgess, J. M., ed. Perspectives in cystic fibrosis: Proceedings of the VIIIth International Cystic Fibrosis Congress. (Canadian Cystic Fibrosis Foundation, Toronto, 1980).

15. Kopel, F. B.: Gastrointestinal manifestations in cystic fibrosis. Gastroenterology, 62: 483 (1972).

16. Kuo, P. T. and Huang, N. N.: The effects of medium chain triglyceride upon fat absorption and plasma lipid and depot fat of children with cystic fibrosis of the pancreas. J. Clin. Invest., 44: 1924 (1965).

17. Lack, L. and Weiner, I. M.: Role of the intestine during the enterohepatic circulation of bile salts. Gastroenterology, 52: 282 (1967).

18. Mangos, J. A. and McSherry, N. R.: Studies on the mechanism of inhibition of sodium transport in cystic fibrosis of the pancreas. Pediatr. Res., 2: 378 (1968).

19. Miettinen, T. A. and Peltokallio, P.: Bile salt, fat, water and vitamin $B_{12}$ excretion after ileostomy. Scan. J. Gastroent., 6: 543 (1971).

20. Milla, P. J., Rassam, U. B., Kilby, A., Ersser, R., and Harries, J. T.: Small intestinal absorption of amino acids and dipeptides in pancreatic insufficiency. In: Sturgess, J. M., ed. Perspectives in cystic fibrosis: Proceedings of the VIIIth International Cystic Fibrosis Congress. (Canadian Cystic Fibrosis Foundation, 
Toronto, 1980).

21. Morin, C. L., Roy, C. C., Lasalle, R., and Bonin, A.: Small bowel mucosal dysfunction in patients with cystic fibrosis. J. Pediatr., 88: 213 (1976).

22. Murphy, G. M., Billing, B. H., and Baron, D. N.: A fluorometric and enzymatic method for the estimation of serum total bile acids. J. Clin. Pathol., 23: 594 (1970).

23. Partin, J. C. and Schubert, W. K.: Small intestinal mucosa in cholesterol ester storage disease. Gastroenterology. 57: 542 (1969).

24. Roy, C. C., Delage, G., Fontaine, A., Robitaille, L., Chartrand, L., Weber, A., and Morin, C. L.: The fecal microflora and bile acids in children with cystic fibrosis. Am. J. Clin. Nutr., 32: 2402 (1979).

25. Rucker, R. W. and Harrison. G. M.: Vitamin $B_{12}$ deficiency in cystic fibrosis. N. Engl. J. Med.. 289: 329 (1973).

26. Scanlin, T. F. and Glick, M. C.: Dynamic and structural aspects of cystic fibrosis fibroblast membranes. In: Sturgess, J. M. ed. Perspectives in cystic fibrosis: Proceedings of the VIIIth International Cystic Fibrosis Congress. (Canadian Cystic Fibrosis Foundation. Toronto, 1980).

27. Thomaidis, T. S. and Arey, J. B.: The intestinal lesions in cystic fibrosis of the pancreas. J. Pediatr., 63: 444 (1963).

28. Torstenson, O. L., Humphrey, G. B., Edson, J. R., and Warwick, W. J.: Cystic fibrosis presenting with severe hemorrhage due to vitamin $\mathrm{K}$ malabsorption: $\mathrm{A}$ report of three cases. Pediatrics, 45: 857 (1970).

29. Tyor, M. P., Garbutt, J. T. and Lack, L.: Metabolism and transport of bile salts in the intestine. Am. J. Med., 51:614 (1971).

Copyright $(1) 1982$ International Pediatric Research Foundation, Inc. $0031-3998 / 82 / 1606-0494 \$ 02.00 / 0$
30. Watkins, J. B.. Tercyak, A. M., Szczepanik, P. and Klein, P. D.: Bile salt kinetics in cystic fibrosis (CF): Influence of pancreatic enzyme replacement. Gastrenterology, 73: 1023 (1977).

31. Weber, A. M., Chartrand, L., Doyon, G., Gordon, S., and Roy, C. C.: Determination of fecal bile acids by the enzymatic method. Clin. Chim. Acta., 39: 524 (1972).

32. Weber, A. M., Roy, C. C., Chartrand, L., Lepage, G., DuFour, O. L., Morin, C. L., and Lasalle, R.: Relationship between bile acid malabsorption and pancreatic insufficiency in cystic fibrosis. Gut, 17: 295 (1976).

33. Weber, A. M., Roy, C. C., Morin, C. L., and Lasalle, R.: Malabsorption of bile acids in children with cystic fibrosis. N. Engl. J. Med., 289: 1001 (1973).

34. The authors are indebted to Dr. Robert Ingberg of the Cystic Fibrosis Clinic, Children's Hospital Medical Center, Cincinnati, Ohio, for his assistance. We wish also to thank Dr. Ralph Giannella for his helpful review of this manuscript and Dr. James A. McAdams for his evaluation of the electron micrographs taken in this study.

35. Requests for reprints should be addressed to: Dr. J. D. Fondacaro, Department of Physiology, University of Cincinnati, College of Medicine, 231 Bethesda Avenue, Cincinnati. OH 45267.

36. This research was supported by NIH grant AM-26650 and by a grant from the Cystic Fibrosis Foundation (JDF) and by NIH grant RR-00123 awarded to the Children's Hospital Clinical Research Center.

37. Received for publication November 24, 1981.

38. Accepted for publication March 15, 1982

Printed in U.S.A. 